\title{
Mucormicosis cutánea primaria: a propósito de dos casos. Revisión de la literatura
}

\author{
Oscar Tapia E., Carolina Chahín A. y Carla Concha F.
}

\section{Primary cutaneous mucormycosis: two case reports and literature}

Mucormycosis or zygomycosis is a rare opportunistic infection caused by aerobic saprophytic fungus that belongs to the class of Zygomycetes Mucorales family. These organisms live in the environment and enter the body by air, gastrointestinal or skin routes, through solutions of continuity of the skin. This microorganism is generally not pathogenic for immunocompetent hosts, being the development of the disease linked with the immune status of the subject. Its mortality is around 50-60\%; sometimes in spite of early diagnosis and treatment initiation it has a fatal course. Six clinical forms of mucormycosis are described: rhinocerebral, cutaneous, pulmonary, disseminated, gastrointestinal and miscellaneous form. Two cases of patients with primary cutaneous mucormycosis diagnosed in the Pathology Unit of Hernan Henriquez Aravena Hospital of Temuco, Chile are presented here.

Key words: Mucormycosis, Zygomycosis, cutaneous mucormycosis.

Palabras clave: Mucormicosis, zigomicosis, mucormicosis cutánea.

\section{Introducción}

L a mucormicosis o zigomicosis corresponde a la tercera infección fúngica invasora más frecuente después de la candidiasis y aspergilosis, reportando series extranjeras una incidencia anual de 1,7 casos/1 millón de habitantes ${ }^{1-3}$. Corresponde a una infección oportunista causada por un hongo saprófito aeróbico que pertenece a la clase Zygomycetes de la familia Mucorales; dentro de esta familia los géneros más frecuentemente aislados son Mucor, Rhizopus, Absidia y Rhizomucor, aunque también pueden ser causadas por otros géneros como Cunninghamella. Rhizopus spp es el agente etiológico más común de mucormicosis en humanos ${ }^{2,4,5}$.

Este agente generalmente no es patógeno para el hospedero inmunocompetente y el desarrollo de la enfermedad está relacionado con el estado inmune del sujeto, encontrándose ampliamente documentado en la literatura científica su frecuente asociación con condiciones tales como cetoacidosis diabética, inmunosupresión farmacológica, inmunodeficiencia adquirida, grandes quemaduras, insuficiencia renal crónica, usuarios de drogas por vía intravenosa y neoplasias hematológicas, aun cuando existen escasos reportes en sujetos inmunocompetentes ${ }^{2,4-9}$.

Se describen seis formas clínicas de mucormicosis: rinocerebral (44-49\%), cutánea (10-16\%), pulmonar (10\%), diseminada (6\%-11\%), gastrointestinal (2\%-11\%) y una forma miscelánea ${ }^{2,4,5,10}$.

$\mathrm{Su}$ mortalidad es cercana a 50-60\%; en ocasiones, a pesar del diagnóstico precoz e instauración del tratamiento específico, tiene un curso fatal ${ }^{2,4,8}$.
Presentamos dos casos de pacientes con mucormicosis cutánea primaria diagnosticados en la Unidad de Anatomía Patológica del Hospital Hernán Henríquez Aravena de Temuco en el año 2009.

\section{Caso 1}

Hombre de 33 años con residencia urbana en la Región de la Araucanía, sin antecedentes mórbidos de importancia, que ingresa al Hospital Dr. Hernán Henríquez Aravena de Temuco por presentar desde una semana antes anorexia, astenia, mialgias generalizadas, cefalea, náuseas y disnea de esfuerzos los últimos tres días, refiriendo haber habitado en un sector rural de la región recientemente. Los exámenes de ingreso mostraban hematocrito de $47 \%$, leucocitos $5.600 / \mathrm{mm}^{3}$ y plaquetas $60.000 / \mathrm{mm}^{3}$ con infiltrados intersticiales difusos bilaterales en la radiografía de tórax. Dado el cuadro clínico y los antecedentes epidemiológicos se planteó un síndrome cardiopulmonar por hantavirus confirmándose la sospecha diagnóstica mediante test rápido específico (inmunocromatografía para IgM). Se inició manejo de soporte e indicó $1 \mathrm{~g}$ /día de metilprednisolona iv durante 5 días. Evolucionó con disfunción miocárdica, requiriendo altas dosis de fármacos vasoactivos. Desarrolló durante su estadía hospitalaria una neumonía asociada a ventilación mecánica tratada inicialmente con imipenem, vancomicina y amikacina. En el cultivo de secreción bronquial se aisló Staphylococcus aureus resistente a meticilina, cambiándose vancomicina por linezolid.
Universidad de La Frontera. Temuco, Chile.

Facultad de Medicina

Departamento de Anatomía Patológica (OTE).

Scientific and Technological Bioresource Nucleus (BIOREN) (OTE).

Hospital Hernán Henríquez Aravena, Temuco, Chile.

Unidad de Infectología (CChA, CCF).

Recibido: 23 de septiembre de 2011

Aceptado: 11 de febrero de 2011

Correspondencia a: Oscar Tapia Escalona otescalona@gmail.com 
Al sexto día de hospitalización inició un cuadro de ictericia progresiva con hiperbilirrubinemia $(13,9 \mathrm{mg} / \mathrm{dL})$ de predominio directa $(12,4 \mathrm{mg} / \mathrm{dL})$, aumento de fosfatasas alcalinas (854 U/1) y una ecotomografía abdominal compatible con una colecistitis aguda alitiásica, la que fue resuelta quirúrgicamente el mismo día.

Durante la segunda y tercera semana, el paciente continuó febril con aparición de lesiones costrosas en el tronco y la cara, una de ellas de localización nasal. Se obtuvieron cultivos de sangre y aspirado endotraqueal, los que fueron informados como positivos para Candida albicans y Pseudomonas aeruginosa respectivamente, agregándose al esquema antimicrobiano cobertura con anidulafungina para C. albicans y cefoperazona/sulbactam para Pseudomonas aeruginosa. Permaneció grave, aunque afebril y, desde el punto de vista cardiopulmonar, requiriendo menor apoyo ventilatorio, con modificación de los parámetros de soporte y de aminas vasoactivas. Durante la cuarta semana de hospitalización se describió la aparición de una lesión ulcerada en el dorso nasal, de fondo necrótico y bordes irregulares (Figura 1a), además de una escara sacra y otras en los talones.

Continuó afebril y en tratamiento con cefoperazona/ sulbactam y anidulafungina. Un cultivo de la lesión nasal fue informado como positivo para C. albicans. En la biopsia de la misma lesión se apreciaban abundantes elementos micóticos tipo hifas no septadas, compatibles con mucor, en un fragmento del ala nasal (Figura 2). Con estos resultados se suspendió la anidulafungina e inició terapia con anfotericina $\mathrm{B}$ desoxicolato en dosis ajustada según función renal (creatininemia: $3 \mathrm{mg} / \mathrm{dL}$ ). El estudio con fondo de ojo, tomografía axial computada (TAC) cerebral y de cavidades paranasales no demostró la concomitancia de lesiones profundas.

Permaneció grave, con ictericia intensa (bilirrubinemia total: $21 \mathrm{mg} / \mathrm{dL}$, bilirrubinemia directa: $19 \mathrm{mg} / \mathrm{dL}$, fosfatasas alcalinas: $854 \mathrm{U} / 1, \mathrm{GOT}: 83 \mathrm{U} / \mathrm{L}, \mathrm{GPT}: 8 \mathrm{U} / \mathrm{L}$ ). La TAC de abdomen-pélvis demostró hepatomegalia difusa sin abscedación, con una vía biliar de estructura

Figura 1. A (caso 1) y $B$ (caso 2). Se aprecian extensas lesiones ulcero-necróticas con depósito de material fibrinopurulento y compromiso profundo de tejidos blandos y óseo. conservada. Una TAC de tórax comprobó la existencia de una extensa efusión pleural a izquierda y el colapso pulmonar ipsilateral. La punción de esta efusión dio salida a líquido hemático que no coagulaba y se instaló un tubo de drenaje pleural.

En los días posteriores evolucionó febril, en anuria, con altos requerimientos de apoyo ventilatorio y hemodinámico. El paciente falleció, habiendo completado cinco días de tratamiento con anfotericina B y luego de efectuarse dos aseos quirúrgicos de la lesión nasal.

\section{Caso 2}

Hombre de 47 años con antecedentes de diabetes mellitus diagnosticada cinco años antes, con irregular control metabólico (amaurosis bilateral y dos hospitalizaciones en el último año por cetoacidosis diabética), y en tratamiento inicialmente con glibenclamida y luego insulina NPH. Consultó por cuadro de dos semanas de evolución caracterizado por dolor y aumento de volumen progresivo de la pierna izquierda secundario a una caída, asociado a somnolencia, anorexia y dificultad respiratoria, sin fiebre. En una centro primario de salud se le administró 4 millones de UI de penicilina G sódica.

A su ingreso al servicio de urgencia se le constató en sopor, afebril, hipotenso y deshidratado. Al examen físico de las extremidades inferiores destacaba una lesión necrótica y flictenas en la zona pretibial izquierda de $30 \mathrm{x}$ $10 \mathrm{~cm}$, ausencia de pulsos poplíteo y pedio a la palpación.

Los exámenes mostraron leucocitosis $\left(20.400 / \mathrm{mm}^{3}\right)$, hiperglicemia (539 mg/dL), acidosis metabólica ( $\mathrm{pH} 6,9)$, proteína $\mathrm{C}$ reactiva elevada $(189 \mathrm{mg} / \mathrm{L})$ y disfunción renal (creatininemia: $1,87 \mathrm{mg} / \mathrm{dL}$, potasio: $5,5 \mathrm{mmol} / \mathrm{L}$ ).

Se le efectuó aseo quirúrgico encontrándose una necrosis muscular del compartimiento antero lateral de la pierna izquierda; se envió muestras para cultivo y biopsia. Ingresó a la UCI con el diagnóstico de fascitis necrosante y sepsis grave, iniciando tratamiento antimicrobiano con ceftriaxona y clindamicina, analgesia con fentanilo y corrección metabólica con bicarbonato de sodio, gluconato de calcio, aporte de volumen e insulina.

Evolucionó hacia la estabilidad, afebril, con mejoría de sus parámetros metabólicos pero con persistencia y progresión de la lesión necrótica en la pierna izquierda. El estudio histopatológico informó la existencia de un tejido fibroconectivo denso y muscular esquelético con un proceso inflamatorio agudo necrosante y presencia de elementos micóticos de tipo hifas no septadas, compatibles con mucor (Figura 2), por lo que se indicó anfotericina $\mathrm{B}$ desoxicolato. El cultivo resultó negativo para elementos micóticos.

Luego de 20 días de tratamiento antifúngico y tres aseos quirúrgicos, la RM evidenciaba un compromiso infeccioso necrosante hasta la tuberosidad de la tibia por 


\section{Caso Clínico}

lo que se decidió realizar amputación suprapatelar de la pierna izquierda (Figura 1b). El estudio con biopsia intraoperatoria del margen de sección proximal no demostró la presencia de elementos micóticos de tipo mucor.

El paciente evolucionó favorablemente desde el punto de vista quirúrgico e infeccioso completando 30 días de tratamiento con anfotericina B. Fue dado de alta encontrándose, al momento de preparar este reporte, bajo controles con fisiatra y terapia ocupacional.

\section{Discusión}

La mucormicosis es una infección oportunista grave que afecta frecuentemente a pacientes con inmunodepresión adquirida o farmacológica y a sujetos diabéticos. Es producida por un hongo saprofito aeróbico que pertenece a la clase Zygomycetes de la familia Mucorales. Estos microorganismos son comúnmente aislados de fuentes ambientales y rara vez producen infección en sujetos inmunocompetentes. Penetran en el organismo por vía aérea, gastrointestinal o cutánea, a través de soluciones de continuidad de la piel ${ }^{1-9}$.

Se reconocen dos formas de mucormicosis cutánea: primaria, con una puerta de entrada exclusivamente cutánea y secundaria, debido a localizaciones metastásicas de una mucormicosis rinocerebral, pulmonar o gastrointestinal. En su forma cutánea primaria representa alrededor de $10 \%$ de los casos de zigomicosis, desarrollándose generalmente en sitios con pérdida de la integridad de la barrera epidérmica (picadura de insecto, contusión, quemadura, inyección intramuscular o venosa, cirugía); en $\sim 70 \%$ de los casos existe el antecedente de una lesión cutánea previa. La asociación de lesión cutánea e inmunodepresión se ha encontrado en $38 \%$ de los casos y en reportes aislados se han visto afectados pacientes inmunocompetentes sin antecedentes de traumatismo ${ }^{2,411-13}$. En ambos casos presentados existían factores predisponentes para la infección, tales como diabetes mellitus descompensada y terapia prolongada con corticoesteroides mientras que en sólo uno de ellos se reconoció una clara puerta de entrada cutánea.

Según la extensión de la lesión y el estado inmune del hospedero se reconocen dos formas de presentación de la mucormicosis cutánea primaria; superficial y gangrenosa o subcutánea; la primera afecta principalmente a sujetos inmunocompetentes, en quienes se desarrolla una lesión necrótica de lenta evolución relativamente circunscrita, mientras que la segunda afecta generalmente a pacientes inmunocomprometidos y/o diabéticos, quienes desarrollan lesiones necróticas extensas, de rápida evolución con compromiso de los planos muscular y óseo ${ }^{4,11,14-16}$. Los dos casos reportados se presentaron en sujetos con patologías predisponentes, asociadas a lesiones necróticas profundas

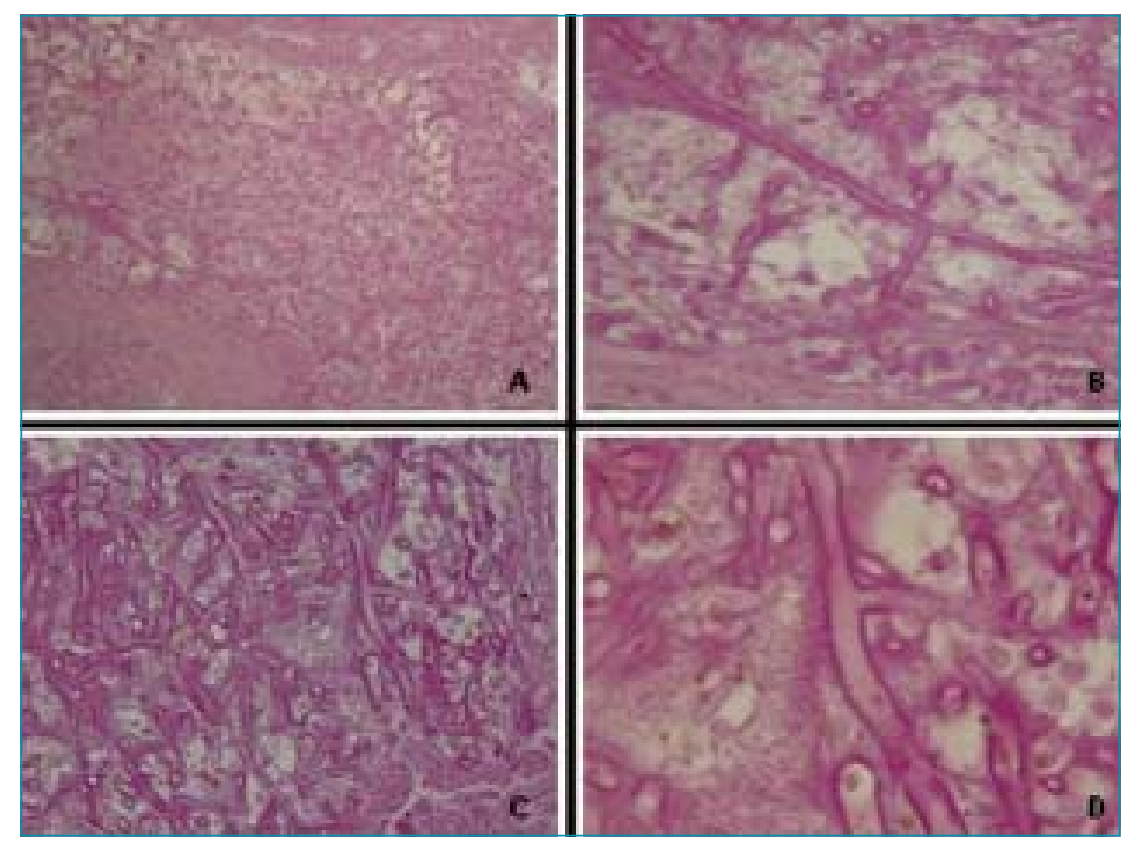

Figura 2. $A(H E, 40 x), B(H E, 100 x), C(P A S, 100 x)$ y D (PAS, 400x). Se observan extensas áreas de tejido necrótico con presencia de abundantes hifas de paredes irregulares, de aspecto ópticamente vacío, que carecen de septos y se ramifican en ángulo recto.

y extensas que comprometían hasta el cartílago del ala nasal y los huesos largos de la pierna, respectivamente, correspondiendo ambos a la forma gangrenosa.

La sospecha clínica no siempre es fácil, pues no existen lesiones cutáneas específicas; pueden presentarse como nódulos o úlceras dolorosas por la celulitis adyacente o indoloras por la extensa necrosis, adoptando en $\sim 65 \%$ de los casos un aspecto necrótico ${ }^{2,4,11}$.

El diagnóstico definitivo de mucormicosis se apoya en el examen directo, el cultivo y la biopsia, siendo de vital importancia pronóstica la precoz sospecha clínica e identificación de esta infección, que permitan instaurar oportunamente el tratamiento etiológico. El examen directo en fresco o preparado con $\mathrm{KOH} 10 \%$ muestra hifas gruesas no septadas con ramificaciones en ángulo recto. El cultivo, por su parte, sirve para precisar la especie, lo que involucra un mayor tiempo y no siempre es positivo. En los casos de esta comunicación, el cultivo resultó negativo- la sensibilidad que se cita para el procedimiento es cercana a $60 \%$-; por el contrario, un cultivo positivo debe analizarse cuidadosamente considerando el cuadro clínico, pues estos agentes pueden ser contaminantes ambientales ${ }^{5,17}$. El examen histopatológico del tejido comprometido demuestra, como en los casos presentados, extensas áreas de necrosis y presencia de hifas con ramificaciones en ángulo recto, ausencia de septos y de paredes irregulares, 
con un ancho entre 15 y $20 \mu \mathrm{m}$, visibles con tinción de hematoxilina-eosina o mediante técnicas histoquímicas especiales tales como Grocott y ácido peryódico de Schiff (PAS) debiéndose plantear el diagnóstico diferencial con otros tipos de hongos filamentosos tales como Aspergillus spp, Fusarium spp y Pseudallescheria boydii. Por otro lado, la tinción fluorescente de calcoflúor ha demostrado su utilidad y rapidez para el diagnóstico tanto en tejidos frescos como fijados; sin embargo, requiere disponer de un microscopio de fluorescencia ${ }^{2,4,10,11,17-20}$.

La terapia para esta infección se basa en tres pilares fundamentales: tratamiento de la enfermedad de base, terapia antifúngica sistémica y cirugía. La terapia antifúngica más ampliamente aceptada es con anfotericina $\mathrm{B}$; el uso de otros fármacos antifúngicos o asociaciones de fármacos con efecto sinérgico es actualmente controversial y aún no está recomendado. Si bien la respuesta terapéutica está limitada por el compromiso inmune del hospedero y la poca penetración del fármaco en un tejido isquémico y con trombosis venosa, anfotericina $\mathrm{B}$ ha permitido mejorar significativamente el pronóstico en los pacientes con mucormicosis ${ }^{2,21}$. La dosis recomendada es 1 a $1,5 \mathrm{mg} / \mathrm{kg} /$ día para alcanzar una dosis total acumulada de 2 a 4 grs, planteando algunos autores la mantención del tratamiento durante 3 a 6 semanas o hasta la remisión de los síntomas. Las suspensiones lipídicas de anfotericina B (llámese liposomal, complejo lipídico o dispersión coloidal) presentan menor toxicidad y mayor difusión en los tejidos, con la consiguiente mejor respuesta clínica. En el primer caso presentando, no disponiendo de formas lipídicas de anfotericina, se ajustó la dosis a emplear de acuerdo a la función renal del paciente, lo que determinó una menor biodisponiblidad del medicamento y un menor efecto fungicida; pudiendo explicar en parte esta variable su desenlace fatal. Otra alternativa terapéutica, hoy en día, está representada por un nuevo azol: posaconazol, el cual ha demostrado buena actividad in vitro frente Rhizopus, Mucor y otras especies de Zygomycetes, existiendo reportes con resultados promisorios en el tratamiento de mucormicosis, tanto en monoterapia o bien como coadyuvante a la terapia con anfotericina; sin embargo, se requiere de mayor evidencia científica que permita validar estos resultados ${ }^{22-27}$.

El tratamiento quirúrgico como pilar en el tratamiento de la mucormicosis cutánea debe ser agresivo y precoz con desbridamiento extenso, remoción de todo el tejido infectado y necrótico, siendo en ocasiones, y como en el segundo caso presentado, necesario amputar la extremidad afectada. Los aseos quirúrgicos deben repetirse en forma periódica ya que disminuyen la carga fúngica y, por tanto, retrasan la progresión de la enfermedad. El acto operatorio debe incluir además la realización de biopsia intraoperatoria de modo de asegurar la obtención de bordes libres de elementos micóticos. Otras alternativas de terapia adyuvante es la oxigenación hiperbárica, la que tendría un efecto fungicida dañando las membranas celulares junto con mejorar la oxigenación y vascularización en la periferia de la lesión ${ }^{2,4,5,8,11}$.

Finalmente, debemos mencionar que la mucormicosis presenta una mortalidad cercana a 50-60\%; en ocasiones, a pesar del precoz diagnóstico e instauración del tratamiento, su curso es fatal, mencionándose en la literatura médica, como factores asociados al pronóstico, la edad, enfermedad de base o condición predisponente para desarrollar la enfermedad, localización de la infección, oportuno diagnóstico e instauración de tratamiento farmacológico y/o quirúrgico que permitan controlar y/o eliminar el agente y los tejidos comprometidos $2,4,8,9,13,28,29$.

En resumen, podemos concluir que la mucormicosis cutánea es una entidad clínico-patológica poco frecuente. El médico clínico debe tener un alto índice de sospecha frente a lesiones cutáneas úlcero-necróticas en pacientes con factores de riesgo de desarrollarla y, de esta manera, plantear un diagnóstico en forma precoz que le permita instaurar un tratamiento oportuno y adecuado.

\section{Resumen}

La mucormicosis o zigomicosis es una infección oportunista poco frecuente, causada por un hongo saprófito aeróbico que pertenece a la clase Zygomycetes de la familia Mucorales. Estos microorganismos viven en el ambiente y penetran en el organismo por vía aérea, gastrointestinal o cutánea a través de soluciones de continuidad de la piel. Este agente generalmente no es patógeno para el hospedero inmunocompetente estando relacionado el desarrollo de la enfermedad con el estado inmune del sujeto. Su mortalidad es cercana a 50-60\%; en ocasiones, a pesar del precoz diagnóstico e instauración del tratamiento, tiene un curso fatal. Se describen seis formas clínicas de mucormicosis: rinocerebral, cutánea, pulmonar, diseminada, gastrointestinal y una forma miscelánea. Se presentan dos casos de pacientes con mucormicosis cutánea primaria diagnosticados en la Unidad de Anatomía Patológica del Hospital Hernán Henríquez Aravena de Temuco. 


\section{Referencias}

1.- $\quad$ Eucker J, Sezer O, Graf B, Possinger K. Mucormycoses. Mycoses 2001; 44: 253-60.

2.- Pak J, Tucci V T, Vincent A L, Sandin R L, Greene J N. Mucormycosis in immunochallenged patients. J Emerg Trauma Shock 2008; 1: 106-113.

3.- Rees J, Pinner R, Hajjeh R, Brandt M, Reingold A. The epidemiological features of invasive mycotic infections in the San Francisco Bay area, 1992-1993: Results of populationbased laboratory active surveillance. Clin Infect Dis 1998; 27: 1138-47.

4.- Spalloni W, Chávez A, Avilés C, Cofré J. Mucormicosis en Pediatría. Rev Chil Infectol 2004; 21: 17-25.

5.- Rahal M, Moreno M, Villa J. Rhino-orbitalcerebral mucormycosis: A twelve-year experience. Rev Otorrinolaringol Cir Cabeza Cuello 2008; 68: 27-34.

6.- Ribes J, Vanover-Sams C, Baker D. Zygomycetes in human disease. Clin Microbiol Rev 2000; 13: 236-301.

7.- Prabhu R, Patel R. Mucormycosis and entomophthoramycosis: a review of the clinical manifestations, diagnosis and treatment Clin Microbiol Infect 2004; 10: 31-47.

8.- Macías J, del Cojo E, Zambrano D, Torrado M, Jiménez J. Estudio descriptivo de cuatro pacientes afectados de mucormicosis ingresados en nuestra unidad de reanimación. Rev Esp Anestesiol Reanim 2004; 51: 385-9.

9.- Geller J, Peters M, Su W. Cutaneous mucormycosis resembling superficial granulomatous pyoderma in an immunocompetent host. J Am Acad Dermatol. 1993; 29: 462-5.

10.- de Mol P, Meis J. Disseminated Rhizopus microsporus infection in a patient on oral corticosteroid treatment: a case report. The Nether J Med 2009, 67: 25-8.

11.- Pérez-Uribe A, Molina de Soschin D, Arenas R,
Reyes M. Mucormicosis cutánea primaria en un paciente con virus de la inmunodeficiencia humana. Rev Iberoam Micol 2005; 22: 118-21.

12.- Loree S, Dompmartin A, Duhamel C, Verneuil L, Comoz D, Leroy D. Mucormycose cutanée primaire. Rapport d'un cas chez un greffé cardiaque et revue de la littérature. J Mycol Med 2001; 11: 44-9.

13.- Adam R, Hunter G, Di Tomasso J, Comerci G Jr. Mucormycosis: Emerging prominence of cutaneous infection. Clin Infec Dis 1994; 19: 67-76.

14.- Oh D, Notrica D. Primary cutaneous mucormycosis in infants and neonates: Case report and review of the literature. J Pediatr Surg 2002; 37: 1607-11.

15.- Scheffler E, Miller G, Classen D. Zygomycosis infection of the neonate upper extremity. J Pediatr Surg 2003; 38: E21.

16.- Ryan M, Ochs D, Ochs J. Primary cutaneous mucormycosis: Superficial and gangrenous infections. Pediatr Infect Dis 1982; 1: 110-14.

17.- Greenberg R, Scott L, Vaughn H, Ribes J. Zygomycosis (mucormycosis): emerging clinical importance and new treatments. Curr Opin Infect Dis 2004; 17: 517-25.

18.- Tarrand J, Lichterfeld M, Warraich I, Luna M, Han X, May G, et al. Diagnosis of invasive septate mold infections: a correlation of microbiological culture and histologic or cytologic examination. Am J Clin Pathol 2003; 119: 854-8.

19.- Frater J, Hall G, Procop G. Histologic features of zygomycosis: Emphasis on perineural invasion and fungal morphology. Arch Pathol Lab Med 2001; 125: 375-8.

20.- García P, Beltrán C, Guzmán A, León P, Arredondo M, Fonseca X. Diagnóstico rápido de dos casos de mucormicosis con tinción de blanco de calcoflúor. Rev Chil Infectol 2001; 18: $285-90$.

21.- Diekema D J, Messer S A, Hollis R J, Jones
R N, Pfaller M A. Activities of caspofungin, posaconazole, ravuconazole, voriconazole and amphotericin B against 448 recent clinical isolates of filamentous fungi. J Clin Microbiol 2003; 41: 3623-6.

22.- Torres H, Hachem R, Chemaly R, Kontoyiannis D, Raad I. Posaconazole: A broadspectrum triazole antifungal. Lancet Infect Dis 2005; 5: 775-85.

23.- Sun Q, Fother hill A, McCarthy D, Rinaldi M, Graybill J. In vitro activities of posaconazole, voriconazole, amphotericin $\mathrm{B}$, and fluconazole against 37 clinical isolates of Zygomycetes. Antimicrob Agents Chemother 2002; 46: 1581-2.

24.- Dannaoui E, Meletiadis J, Mouton J, Meis J, Verwirj P. The Eurofung Network. In vitro susceptibilities of zygomycetes to conventional and new antifungals. J Antimicrob Chemother 2003; 51: 45-52.

25.- Tobon A, Arango M, Fernández D, Restrepo A. Mucormycosis (zygomycosis) in a heartkidney transplant recipient: recovery after posaconazole therapy. Clin Infect Dis 2003; 36: $1488-91$

26.- Pfaller M, Mecer S, Boyken L, Hollis R, Diekema D. Posaconazole is a new triazole with in vitro and in vivo activity against Zygomicosis. Diag Microbiol Infect Dis 2003; 45: 241-4.

27.- Seguin P, Musellec H, Le Gall F, Chevrier S, Le Bouquin V, Malledant Y. Post traumatic course complicated by cutaneous infection with Absidia corymbifera. Eur J Clin Microbiol Infect Dis 1999; 18: 737-9.

28.- Roden M, Zaoutis T, Buchanan W, Knudsen T, Sarkisova T, Schaufele R, et al. Epidemiology and outcome of zygomycosis: a review of 929 reported cases. Clin Infect Dis 2005; 41: 634-53.

29.- Hamilton J, Bartkowski H, Rock J. Management of CNS mucormycosis in the pediatric patient. Pediatr Neurosurg 2003; 38: 212-5. 